\title{
Electron temperature anisotropy instabilities represented by superposition of streams
}

A. Inglebert, A. Ghizzo, T. Reveille, P. Bertrand, and F. Califano

Citation: Phys. Plasmas 19, 122109 (2012); doi: 10.1063/1.4772770

View online: http://dx.doi.org/10.1063/1.4772770

View Table of Contents: http://pop.aip.org/resource/1/PHPAEN/v19/i12

Published by the American Institute of Physics.

\section{Related Articles}

Modulation instability of an intense laser beam in the hot magnetized electron-positron plasma in the quasineutral limit

Phys. Plasmas 19, 122107 (2012)

Global gyrokinetic particle-in-cell simulations of internal kink instabilities

Phys. Plasmas 19, 122104 (2012)

Plasma instability in the afterglow of electron cyclotron resonance discharge sustained in a mirror trap

Phys. Plasmas 19, 122501 (2012)

Spin effect on parametric interactions of waves in magnetoplasmas

Phys. Plasmas 19, 112114 (2012)

Non-linear magnetohydrodynamic simulations of density evolution in Tore Supra sawtoothing plasmas

Phys. Plasmas 19, 112305 (2012)

\section{Additional information on Phys. Plasmas}

Journal Homepage: http://pop.aip.org/

Journal Information: http://pop.aip.org/about/about_the_journal

Top downloads: http://pop.aip.org/features/most_downloaded

Information for Authors: http://pop.aip.org/authors

\section{ADVERTISEMENT}

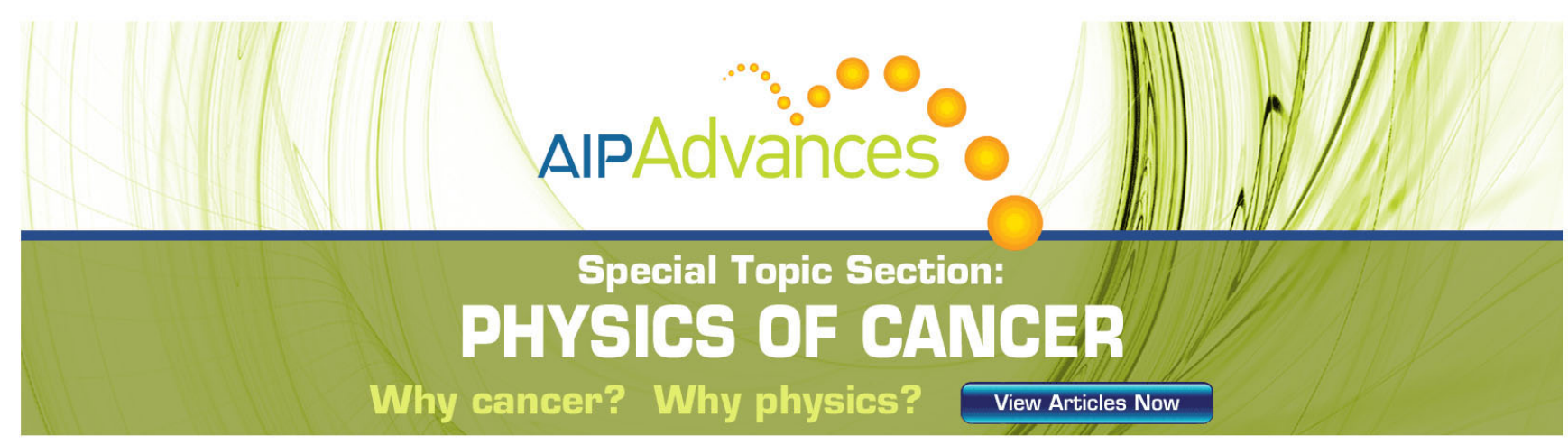




\title{
Electron temperature anisotropy instabilities represented by superposition of streams
}

\author{
A. Inglebert, ${ }^{1}$ A. Ghizzo, ${ }^{1}$ T. Reveille, ${ }^{1}$ P. Bertrand,${ }^{1}$ and F. Califano ${ }^{2}$ \\ ${ }^{1}$ IJL UMR 7198, Université de Lorraine, BP 70239 F-54506 Vandoeuvre les Nancy, France \\ ${ }^{2}$ Department of Physics, University of Pisa, Pisa, Italy
}

(Received 9 October 2012; accepted 29 November 2012; published online 19 December 2012)

The generation of magnetic field, together with the electrostatic activity met in the saturation regime of the Weibel instability (WI), is investigated by means of an analytical multi-stream model in a Hamiltonian framework. Taking advantage from the invariance of the generalized canonical momentum, the model allows to reduce the full kinetic 1D2V Vlasov equation into several $1 \mathrm{D} 1 \mathrm{~V}$ equations while keeping its kinetic character. The multi-stream model provides a more complete and accurate picture of the Weibel instability, because it is possible to separate the specific contribution of each stream during the development of the Weibel instability. An interesting result for the multi-stream mode is a lower cost in the perpendicular treatment of the $p_{y}$ momentum component since no differential operator associated with some approximate numerical scheme has to be carried out on this variable. Indeed, a small number of streams or particle classes are sufficient to correctly describe the magnetic field generation and the mixed electrostatic- electromagnetic nature of the instability. (C) 2012 American Institute of Physics. [http://dx.doi.org/10.1063/1.4772770]

\section{INTRODUCTION}

Electron temperature anisotropy instability, better known as the Weibel instability ${ }^{1}$ (WI) is a fundamental process in space and laboratory plasmas, being the only mechanism able to generate magnetic fields in space and laboratory experiments, in particular in the context of laser-plasma interaction. Starting from an initial temperature anisotropy, a condition that can be realized in non collisional high temperature and/or rarified laboratory and space plasmas, the WI generates "quasi-static" magnetic fields through the redistribution of currents in space. These currents, rearranged through the growing waves into filaments, are separated more and more by the electromagnetic fields thus self-consistently generating a corresponding magnetic field. ${ }^{2}$ Due to the intrinsic kinetic character of this instability, one must make use of the full Vlasov-Maxwell system, which is mathematically and computationally very complex to solve. Any possibility to reduce such complexity, but not limited to the linear regime, is, therefore, of very strong importance for applicative purposes.

The WI emerges from a wide white noise spectrum of wavelengths around the electron skin depth $d_{e}=c / \omega_{p}$. Due to the electromagnetic nature of the instability, the study of the WI was mainly focused on the kinetic saturation process and in particular on the amplitude value and persistence in time of the magnetic field. However, understanding the coupling of the magnetic field with the plasma motions and the associated wave-particle interactions is a challenging issue both from a fundamental and an applied point of view. In this perspective, the understanding of the "electrostatic" activity is of particularly relevance since, even in the onedimensional limit, the electrostatic field plays a key role, at least starting from the saturation phase.

Numerical investigation of electrostatic activity in the saturation regime of the WI has been recently performed by
Stockem et al. ${ }^{3}$ by means of particle-in-cell (PIC) simulations showing the formation of bipolar structures, and by Palodhi et al. ${ }^{4}$ using Eulerian Vlasov simulations showing the occurrence of strong deformations of the electron distribution function (hereafter df) in phase space leading to the generation of short wavelength Langmuir modes. By including a "small" background magnetic field leading to a transition from the WI to the whistler instability, Palodhi et al. ${ }^{5}$ have shown the formation of electrostatic wave packet in the non linear regime, the so-called oscillotons.

The coming into play of the electrostatic field has a double explanation. On one side, on large scales, the non linear coupling of the instability to plasma waves generates an electrostatic field rapidly growing on the same scales of the WI. ${ }^{6}$ On the other hand, the distortion and stretching of the d.f. by the self-generated magnetic field, leads to the formation of local bumps and thus to the set up of a secondary bump on tail instability responsible for the growth of resonant electrostatic modes (ESs) on scales now smaller than the instability length scale. ${ }^{4}$

More recently, using a Hamiltonian description of particle motion and 1D3V PIC simulation, Innocenti et al. ${ }^{7}$ in a similar regime have identified the role of such electrostatic activity as a result of "a two-stream" like instability, leading to the formation of phase space electron holes. These 1D3V PIC simulations show that the Hamiltonian description of particle motion in the 1D limit is a valuable tool to understand the plasma dynamics as described in terms of particle bunches, each bunch having the same perpendicular canonical momentum. Furthermore, the stability of such electron holes were also studied analytically and numerically in previous works ${ }^{8,9}$ showing that long-lived excitations at frequencies at something like a third of the plasma frequency can indeed be produced (if coherently driven up to selfsustaining level) over a wide range of frequencies for a given wavenumber. 
All these works, using an Eulerian or Lagrangian numerical approach, are based on a full kinetic Vlasov model showing that the behavior of the electron d.f. in the $x-p_{y}$ space can be view in terms of a multi-layer type function. In particular, the temperature anisotropy driven Weibel instability initially gives rises to a Y-shaped distribution function, which yields, through its current redistribution, to the growth of the magnetic field. Once the electromagnetic fields become strong, particles fluctuating at nearly the thermal velocity can be accelerated yielding to a layered structure in the $x$ - $p_{y}$ phase space. Thus, the growing magnetic fields modify the particle trajectories, and the saturation of the Weibel instability sets in through electromagnetic trapping.

In this paper, we extend such analysis by investigating the longitudinal and transverse particle dynamics of the WI by means of a kinetic multi-stream model based on a Hamiltonian reduction technique ${ }^{10}$ and by direct $1 \mathrm{D} 2 \mathrm{~V}$ semi-Lagrangian Vlasov-Maxwell simulations. In the one-dimensional limit, this model exploits the exact canonical invariance in the perpendicular direction allowing us to reduce the dimension of the momentum space. The use of such invariants allows us to find out a broad class of exact non linear solutions of the Vlasov-Maxwell system, used to reconstruct the global phase space dynamics.

The paper is organized as follows. In Sec. II, we present the main equations of our multi-stream model as derived from the Vlasov-Maxwell system. Then, we recover the standard dispersion relation of WI in Sec. III. The notion of threshold of WI is recovered by introducing a water-bag description in the longitudinal direction in momentum in the case of temperature anisotropy. Section IV is devoted to the numerical version of the multi-stream model and test validation of the dispersion relation. Numerical comparison of our model with a standard semi-lagrangian 1D2V Vlasov code is carried out in Sec. V. Finally, Sec. VI is reserved for the conclusions and discussions.

\section{BASIC EQUATIONS}

Our model is based on a full kinetic description of the dynamics in the longitudinal direction (here $p_{x}$ ), while a Hamiltonian reduction is used to select a class of exact solutions to represent the dynamics in the perpendicular direction in terms of a sum of particles "bunches" or "streams."

We consider a collisionless plasma where the ions are considered just as a fixed neutralizing background. For the electrons, we must solve the Vlasov equation in phase space to evolve in time the distribution function $F(\boldsymbol{q}, \boldsymbol{p}, t)$

$$
\frac{\partial F}{\partial t}+\frac{\boldsymbol{p}}{m_{e} \gamma} \frac{\partial F}{\partial \boldsymbol{q}}+e\left(\boldsymbol{E}+\frac{\boldsymbol{p} \times \boldsymbol{B}}{m_{e} \gamma}\right) \frac{\partial F}{\partial \boldsymbol{p}}=0,
$$

where $\gamma=\left[1+\boldsymbol{p}^{2} / m_{e}^{2} c^{2}\right]^{1 / 2}$ is the Lorentz factor. The Vlasov Eq. (1) is self-consistently coupled to Maxwell's equations. When the characteristics of the Vlasov equation possess an exact invariant, it is possible to reduce the dimension of the phase space. In particular, in Ref. 10, we have shown that such reduced dynamical description can be expressed in terms of $N$ "streams" or bunches of particles each one satisfying a reduced kinetic Vlasov-type equation independent on the perpendicular kinetic momentum coordinate $\boldsymbol{p}_{\perp}$. The Hamiltonian reduction technique is based on the introduction of the canonical momentum $\boldsymbol{P}_{c}=\boldsymbol{p}+\boldsymbol{e} \boldsymbol{A}_{\perp}$ and on its invariance in the perpendicular direction, $d \boldsymbol{P}_{c, \perp} / d t=0$. Therefore, we can represent the plasma evolution by a class of initial conditions invariant under the dynamics. Without loss of generality, we can consider a plasma represented by $2 N+1$ "streams" of particles, each one labeled by the index $j$ (with $-N \leq j \leq N$ ) having the same initial perpendicular momentum, say $\boldsymbol{P}_{c, \perp}=\boldsymbol{C}_{j}=$ const. Following Ref. 11, we can define, for each particle population $j$, a distribution function $f_{j}\left(x, p_{x}, t\right)$ satisfying a relativistic Vlasov-type equation given by

$$
\frac{\partial f_{j}}{\partial t}+\frac{p_{x}}{m_{e} \gamma_{j}} \frac{\partial f_{j}}{\partial x}+\left(e E_{x}-\frac{1}{2 m_{e} \gamma_{j}} \frac{\partial}{\partial x}\left(\boldsymbol{C}_{j}-e \boldsymbol{A}_{\perp}\right)^{2}\right) \frac{\partial f_{j}}{\partial p_{x}}=0
$$

where the $\gamma_{j}$ Lorentz factor now reads

$$
\gamma_{j}=\left[1+\frac{p_{x}^{2}}{m_{e}^{2} c^{2}}+\frac{\left(\boldsymbol{C}_{j}-e \boldsymbol{A}_{\perp}(x, t)\right)^{2}}{m_{e}^{2} c^{2}}\right]^{1 / 2} .
$$

For each population $j$, we now define the stream density as $n_{j}=\int f_{j} d p_{x} \quad$ and a current density $\boldsymbol{J}_{\perp j}=\left(e / m_{e}\right)$ $\left(\boldsymbol{C}_{j}-e \boldsymbol{A}_{\perp}\right) \int_{-\infty}^{+\infty} f_{j} / \gamma_{j} d p_{x}$. Finally, the reduced Vlasov equations given by Eq. (2) are self-consistently coupled to the equation for the longitudinal electric field $E_{x}$

$$
\frac{\partial E_{x}}{\partial x}=\frac{e}{\varepsilon_{0}}\left(\sum_{j=-N}^{N} n_{j}(x, t)-n_{0}\right)
$$

and to the one for potential vector $\boldsymbol{A}_{\perp}$,

$$
\frac{\partial^{2} \boldsymbol{A}_{\perp}}{\partial t^{2}}-c^{2} \frac{\partial^{2} \boldsymbol{A}_{\perp}}{\partial x^{2}}=\frac{1}{\varepsilon_{0}} \sum_{j=-N}^{N} \boldsymbol{J}_{\perp j}
$$

allowing to reduce the phase space to $2 \mathrm{D}$ one, $\left(x, p_{x}\right)$; each stream being described by a $f_{j}$ distribution function.

\section{APPLICATION TO THE STUDY OF THE WI}

In the $1 \mathrm{D}-2 \mathrm{~V}$ limit, we can write the d.f. $F\left(x, p_{x}, p_{y}, t\right)$ as follows:

$$
F\left(x, p_{x}, p_{y}, t\right)=\sum_{j=-N}^{N} f_{j}\left(x, p_{x}, t\right) \delta\left(p_{y}-\left(C_{j}-e A_{y}(x, t)\right)\right)
$$

thus reducing the $3 D\left(x, p_{x}, p_{y}\right)$ phase space into a $2 \mathrm{D}\left(x-p_{x}\right)$ one plus $2 N+1$ values of the corresponding $C_{j}$. Here, we consider a linearly polarized electromagnetic wave and choose $\boldsymbol{A}_{\perp}=A_{y} \boldsymbol{e}_{y}$ and $\boldsymbol{P}_{c \perp}=P_{c y} \boldsymbol{e}_{y}=$ const $=C_{j} \boldsymbol{e}_{y}$. Finally, we assume an initially homogeneous plasma. 


\section{A. The multi-stream model and moments of a continu- ous distribution function}

Because we want to study the WI driven by a temperature anisotropy, we need to precise the meaning of "temperature" in our model. In the $p_{x}$ direction, where we keep a full Vlasov description, the temperature is described in a standard way using the data of the d.f. Now, in the $p_{y}$ direction, the concept of temperature may be recovered in the multi-stream model by considering the moments of the d.f. or, in other words, by looking at the equivalence in the fluid momentum sense of the multi-stream distribution and a continuous distribution. To this purpose, let us now consider a Maxwellian equilibrium $F_{M}\left(p_{x}, p_{y}\right)$ of thermal velocity $v_{t h, x}$ and $v_{t h, y}$, and of mean density $n_{0}=1$. By defining $h\left(p_{y}\right)=\int F_{M}\left(p_{x}, p_{y}\right) d p_{x}$, we can use for our multi-stream model the following functional form:

$$
h\left(p_{y}\right)=\sum_{j=-N}^{N} F_{j} \delta\left(p_{y}-C_{j}\right) .
$$

We then define the $2 n-$ moment of $h\left(p_{y}\right)$ as

$$
\int_{-\infty}^{+\infty} p_{y}^{2 n} h\left(p_{y}\right) d p_{y}=\sum_{j=-N}^{N} C_{j}^{2 n} F_{j}
$$

and we take a Maxwellian equilibrium of the type $F_{M}$ $=\left(\sqrt{\alpha_{x} \alpha_{y}} / \pi\right) e^{-\alpha_{y} p_{y}^{2}} e^{-\alpha_{x} p_{x}^{2}}$ with $\alpha_{x / y}=1 / 2 m_{e} k_{B} T_{x / y}$. The equivalence condition with the $2 n$ moments of the distribution $F_{M}$, Eq. (8), gives

$$
\sum_{j=-N}^{N} C_{j}^{2 n} F_{j}=\sqrt{\frac{\alpha_{y}}{\pi}} \int_{-\infty}^{+\infty} p_{y}^{2 n} e^{-\alpha_{y} p_{y}^{2}} d p_{y}=\frac{(2 n-1) ! !}{\left(2 \alpha_{y}\right)^{n}} .
$$

By assuming that the $j$-stream verifies the symmetry properties $C_{-j}=-C_{j}$ and $F_{-j}=F_{j}, j=1, \ldots, N$ and $C_{0}=0$, Eq. (9) gives

$$
2 \sum_{j=1}^{N} C_{j}^{2 n} F_{j}=\frac{(2 n-1) ! !}{\left(2 \alpha_{y}\right)^{n}} .
$$

This equation, together with the normalization condition $\sum_{j} F_{j}=1$, allows us to prepare initially the plasma in the form of an equilibrium. Let us consider, as a first example, the case of a system made by three streams. We must solve the following system:

$$
F_{0}+2 F_{1}=1 ; \quad 2 C_{1}^{2} F_{1}=m_{e}^{2} v_{t h, y}^{2} ; 2 C_{1}^{4} F_{1}=3 m_{e}^{4} v_{t h, y}^{4},
$$

from which we get $F_{0}=\frac{2}{3}, F_{-1}=F_{1}=\frac{1}{6}$, and $C_{-1}=-C_{1}$ $=-\sqrt{3} m_{e} v_{t h, y}$.

However, in the general case, Eq. (10) has the form of a Vandermonde system. In particular, when trying to solve this system numerically, it becomes ill-conditioned for large values of the number of streams. A more convenient way can be found to solve Eq. (10) for a "large" number of streams and for a regular sampling of the $p_{y}$ axis, i.e., for a equispaced set of $C_{j}$ values. The idea is to compute the $F_{j}$ values at points
$C_{j}=j \Delta C$ (using for instance $\Delta C \sim v_{t h, y}$ ) for a given distribution function. Note that the method can be applied for any distribution function even in the relativistic regime. Such an approximation becomes accurate for a total number of streams greater than five. Of course, for a number of streams smaller than, or equal to five, the exact $F_{j}$ can be obtained solving directly the linear system (10). A similar technique has been used in the gyrokinetic modeling using a multiple water-bag approach, where the same problem arises when one has to determine initially the contours of distribution function (for more details see, Ref. 12).

The last point concerns the introduction of the waterbag description in the longitudinal direction. Although our model takes into account a continuous distribution in $p_{x}$, we find more convenient to introduce a simplification in the description of the kinetic effects arising along this direction. Indeed, the water-bag model offers a very good description of the plasma dynamics even with a small number of bags, and opens to analytical investigations. We underline that the water-bag model offers an excellent bridge between the hydrodynamical description and the full Vlasov one.

\section{B. The multi-stream multi water-bag model}

We introduce a water-bag description ${ }^{13,14}$ in the longitudinal momentum direction, $p_{x}$, but still taking a non zero longitudinal temperature $T_{x}$. The initial equilibrium distribution function can be written in the form

$F_{M}\left(p_{x}, p_{y}\right)=\sum_{j=-N}^{+N} G_{j}\left[\mathcal{H}\left(p_{x}+p_{a j}\right)-\mathcal{H}\left(p_{x}-p_{a j}\right)\right] \delta\left(p_{y}-C_{j}\right)$,

where $\mathcal{H}$ is the Heaviside function and the distribution function takes the value $G_{j}$ for $p_{x}$ in the range $\left[-p_{a j}, p_{a j}\right]$ and zero otherwise, $p_{a j}$ being assigned at $t=0$. By calculating the mean value along $p_{y}$,

$$
\begin{aligned}
g\left(p_{x}\right) & =\int_{-\infty}^{+\infty} F_{M}\left(p_{x}, p_{y}\right) d p_{y} \\
& =\sum_{j=-N}^{+N} G_{j}\left[\mathcal{H}\left(p_{x}+p_{a j}\right)-\mathcal{H}\left(p_{x}-p_{a j}\right)\right],
\end{aligned}
$$

and by using the normalization condition $\int g\left(p_{x}\right) d p_{x}=n_{0}$, we get $\sum_{j} 2 p_{a j} G_{j} / n_{0}=1$. Furthermore, the first moment must be zero, $\int p_{x} g\left(p_{x}\right) d p_{x}=0$, and the squared longitudinal thermal momentum is given by

$$
p_{t h, x}^{2}=m_{e}^{2} v_{t h, x}^{2}=\frac{1}{n_{0}} \int_{-\infty}^{+\infty} p_{x}^{2} g\left(p_{x}\right) d p_{x}=\frac{1}{3} \sum_{j=-N}^{N} \alpha_{j} p_{a j}^{2},
$$

where $\alpha_{j}=2 p_{a j} G_{j} / n_{0}$.

The integration over the $p_{x}$ coordinate leads to the definition of the mean distribution function (given previously in Eq. (7)) as

$$
h\left(p_{y}\right)=\int_{-\infty}^{+\infty} F_{M}\left(p_{x}, p_{y}\right) d p_{x}=n_{0} \sum_{j=-N}^{+N} \alpha_{j} \delta\left(p_{y}-C_{j}\right) .
$$


From the condition $\int h\left(p_{y}\right) d p_{y}=n_{0}$, we recover the normalization condition $\sum_{j} \alpha_{j}=1$, met above previously, while the condition $\int p_{y} h\left(p_{y}\right) d p_{y}=0$ leads to $\sum_{j} \alpha_{j} C_{j}=0$. The transverse "thermal" momentum is then obtained from the 2nd order moment as

$$
p_{t h, y}^{2}=m_{e}^{2} v_{t h, y}^{2}=\frac{1}{n_{0}} \int_{-\infty}^{+\infty} p_{y}^{2} h\left(p_{y}\right) d p_{y}=\sum_{j=-N}^{N} \alpha_{j} C_{j}^{2} .
$$

Thus, the equilibrium function is characterized by the set of parameters $\left\{\alpha_{j}, p_{a j}, C_{j}\right\}$, with $-N \leq j \leq N$. The ensemble $\left\{\alpha_{j}, p_{a j}, C_{j}\right\}$ is constant in time and the distribution function $F\left(x, p_{x}, p_{y}, t\right)$ can be written as follows:

$F=\sum_{j=-N}^{+N} G_{j}\left[\mathcal{H}\left(p_{x}-p_{j}^{-}\right)-\mathcal{H}\left(p_{x}-p_{j}^{+}\right)\right] \delta\left(p_{y}-\left(C_{j}-e A_{y}\right)\right)$,

where $p_{j}^{ \pm}$depend on $x$ and $t$. In a water-bag approach, it is sufficient to follow the dynamics of the contours, here obtained by calculating the moments of the reduced Vlasov Eq. (2)

$$
\frac{\partial p_{j}^{ \pm}}{\partial t}+\frac{p_{j}^{ \pm}}{m_{e} \gamma_{j}^{ \pm}} \frac{\partial p_{j}^{ \pm}}{\partial x}=e E_{x}-\frac{1}{2 m_{e} \gamma_{j}^{ \pm}} \frac{\partial}{\partial x}\left(C_{j}-e A_{y}\right)^{2},
$$

self-consistently coupled to the longitudinal electric field $E_{x}$ given by Eq. (4) and to the potential vector $A_{y}$ given by Eq. (5). Finally, $\gamma_{j}^{ \pm}$is the Lorentz factor for the $j$-stream

$$
\gamma_{j}^{ \pm}=\left[1+\frac{p_{j}^{ \pm 2}}{m_{e}^{2} c^{2}}+\frac{\left(C_{j}-e A_{y}(x, t)\right)^{2}}{m_{e}^{2} c^{2}}\right]^{1 / 2} .
$$

Using the definition for the d.f. given in Eq. (17), the equations for the fields $E_{x}$ and $A_{y}$ become

$$
\begin{gathered}
\frac{\partial E_{x}}{\partial x}=\frac{e}{\varepsilon_{0}}\left(\sum_{j=-N}^{N} G_{j}\left(p_{j}^{+}-p_{j}^{-}\right)-n_{0}\right), \\
\frac{\partial^{2} A_{y}}{\partial t^{2}}-c^{2} \frac{\partial^{2} A_{y}}{\partial x^{2}}+\sum_{j=-N}^{N} \omega_{p j}^{2} A_{y}=\frac{1}{e} \sum_{j=-N}^{N} \omega_{p j}^{2} C_{j},
\end{gathered}
$$

where $\omega_{p j}^{2}$ is the squared plasma frequency associated to the $j$-stream

$$
\omega_{p j}^{2}=\frac{e^{2} G_{j}}{m_{e} \varepsilon_{0}} \int_{p_{j}^{-}}^{p_{j}+} \frac{d p_{x}}{\gamma_{j}}
$$

and $\gamma_{j}=\left[1+\frac{p_{x}^{2}}{m_{e}^{2} c^{2}}+\frac{\left(C_{j}-e A_{y}(x, t)\right)^{2}}{m_{e}^{2} c^{2}}\right]^{1 / 2}$ is obtained using Eq. (3).

We consider now the non relativistic limit given by $\gamma_{j} \simeq \gamma_{j}^{ \pm} \simeq 1$ in Eqs. (18), (21), and (22). We now make a linear normal mode analysis assuming for the $j$-stream a mean density $n_{0 j}$ and a contour value $\pm p_{a j}$. Denoting the generic $z$ fluctuating quantity as $\delta z$, and using $p_{j}^{ \pm}= \pm p_{a j}+\delta p_{j}^{ \pm}$ $= \pm m_{e} a_{j}+\delta p_{j}^{ \pm}, n_{j}=n_{0 j}+\delta n_{j}$, Eqs. (18), (20), and (21) become

$$
\begin{gathered}
\frac{\partial \delta p_{j}^{ \pm}}{\partial t} \pm a_{j} \frac{\partial \delta p_{j}^{ \pm}}{\partial x}=e \delta E_{x}+\frac{e C_{j}}{m_{e}} \frac{\partial \delta A_{y}}{\partial x} \\
\frac{\partial \delta E_{x}}{\partial x}=\frac{e}{\varepsilon_{0}} \sum_{j=-N}^{N} G_{j}\left(\delta p_{j}^{+}-\delta p_{j}^{-}\right), \\
\frac{\partial^{2} \delta A_{y}}{\partial t^{2}}-c^{2} \frac{\partial^{2} \delta A_{y}}{\partial x^{2}}+\omega_{p}^{2} \delta A_{y}=\frac{e}{m_{e} \varepsilon_{0}} \sum_{j=-N}^{N} G_{j} C_{j}\left(\delta p_{j}^{+}-\delta p_{j}^{-}\right) .
\end{gathered}
$$

By linearizing these equations using the standard notation $e^{i(k x-\omega t)}$, we get the dispersion relation for the Weibel instability

$$
\begin{gathered}
\left(\omega^{2}-\omega_{k}^{2}-k^{2} \omega_{p}^{2} \sum_{j=-N}^{+N} \frac{\alpha_{j} C_{j}^{2}}{m_{e}^{2}\left(\omega^{2}-k^{2} a_{j}^{2}\right)}\right) \\
\times\left(1-\omega_{p}^{2} \sum_{j=-N}^{+N} \frac{\alpha_{j}}{\omega^{2}-k^{2} a_{j}^{2}}\right) \\
=k^{2} \omega_{p}^{4}\left(\sum_{j=-N}^{+N} \frac{\alpha_{j} C_{j}}{m_{e}\left(\omega^{2}-k^{2} a_{j}^{2}\right)}\right)^{2},
\end{gathered}
$$

where $\omega_{k}^{2}=\omega_{p}^{2}+k^{2} c^{2}$. First of all, in the limit of only one stream, corresponding to $C_{j}=0$ and $a_{j}=a$ for the stream $j=0$, we obtain two independent modes

(i) An electrostatic mode (ES) (with $\delta E_{x} \neq 0$ and $\left.\delta A_{y}=0\right)$ that obeys the standard Bohm-Gross dispersion relation which, in the usual water-bag model, writes as $\omega^{2}=\omega_{p}^{2}+k^{2} a^{2}$. The connection with the Bohm-Gross case is then obtained with the choice $a=\sqrt{3} v_{t h, x}$, where $v_{t h, x}=\sqrt{k_{B} T_{x} / m_{e}}$ is the thermal velocity along $p_{x}$.

(ii) An electromagnetic mode (EM) in the cold plasma with $\omega^{2}=\omega_{k}^{2}$.

Second, in the case of $2 N+1$ streams, and assuming $a_{j}=a$ for any $j$, we recover again two independent ES and EM modes. Indeed, since $\sum_{j} C_{j} \alpha_{j}=0$, the right-hand side of Eq. (26) is zero, so that the ES and EM modes are decoupled. The EM mode, which include the WI of electromagnetic nature, is given by

$$
\omega^{2}-\omega_{k}^{2}-\frac{k^{2} \omega_{p}^{2}}{m_{e}^{2}\left(\omega^{2}-k^{2} a^{2}\right)} \sum_{j=-N}^{+N} \alpha_{j} C_{j}^{2}=0 .
$$

Using the definition of $p_{t h, x}$ and $p_{t h, y}$ and Eqs. (14) and (16), respectively, Eq. (27) becomes

$$
\omega^{2}-\omega_{k}^{2}-\frac{k^{2} \omega_{p}^{2} v_{t h, y}^{2}}{\omega^{2}-3 k^{2} v_{t h, x}^{2}}=0 .
$$

In the cold limit, $v_{t h, y}=0$, we immediately recover the dispersion relation of an EM wave in a cold plasma. The case $v_{t h, y} \neq 0$, instead, corresponds to the standard Weibel 


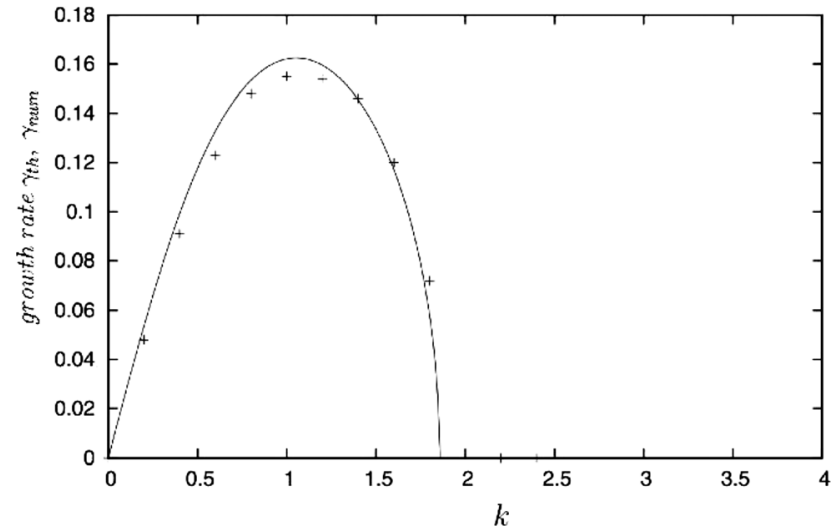

FIG. 1. The growth rate of the WI vs. the wave vector $k$. The continuous line is obtained by solving the dispersion relation, Eq. (29). The crosses are instead evaluated from a numerical simulation of the multi-stream R-model.
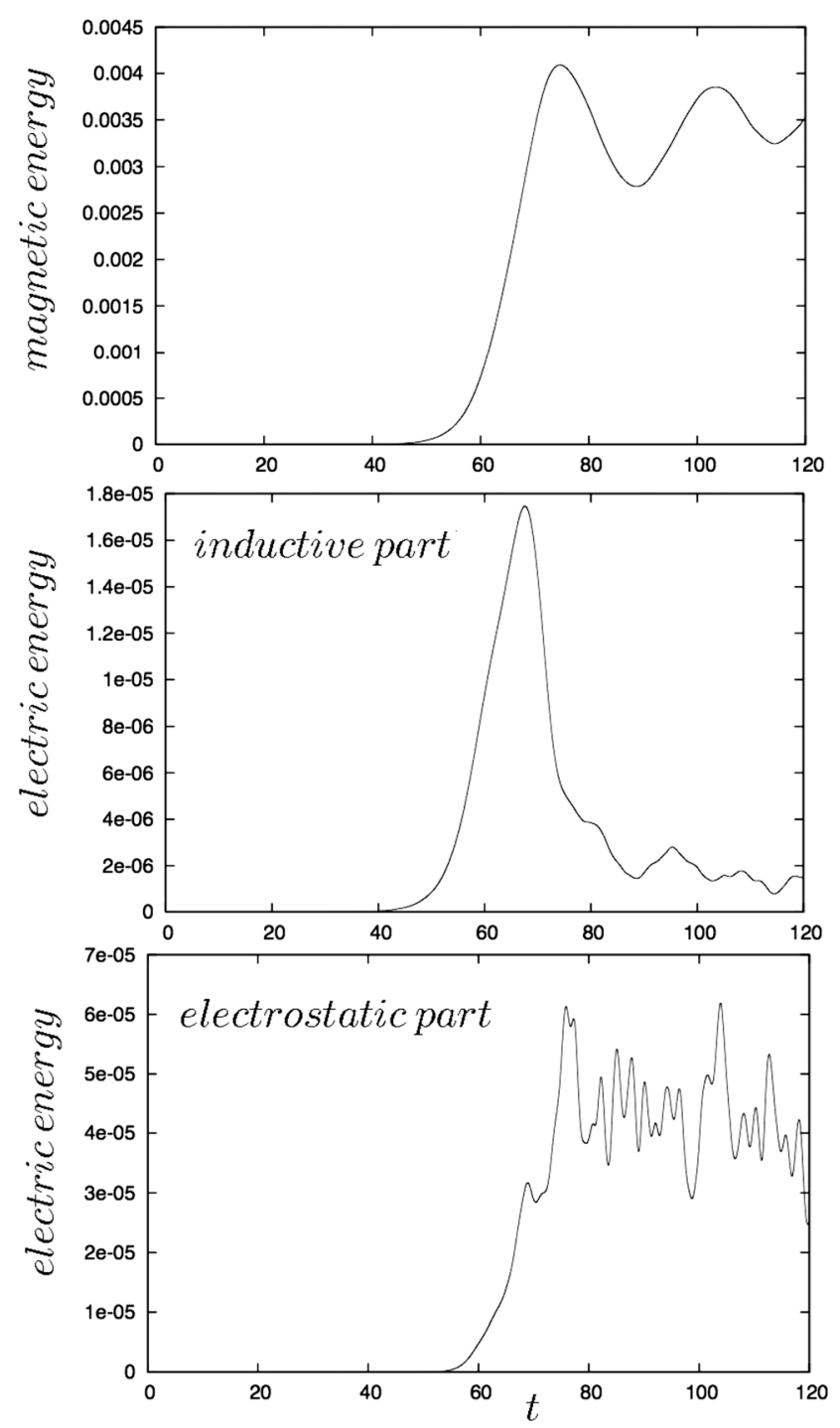

FIG. 2. Time evolution of the magnetic energy, the inductive electric energy and the electrostatic energy, and the top, middle, and bottom panels, respectively. These results represent the evolution of the WI obtained the R-model using five streams and an initial water-bag type distribution function. The initial temperatures are $T_{e x}=2.25 \mathrm{keV}$ and $T_{e y}=50 \mathrm{keV}$. instability, driven by a temperature anisotropy. Equation (28) can be written in a polynomial form of fourth degree given by

$$
\omega^{4}-\left(1+k^{2}+3 k^{2} v_{t h, x}^{2}\right) \omega^{2}+3 k^{2} v_{t h, x}^{2}\left(1+k^{2}\right)-k^{2} v_{t h, y}^{2}=0,
$$

where the frequency, wave vector, and velocity are normalized to the plasma frequency $\omega_{p}$, the electron skin depth $d_{e}=c / \omega_{p}$, and the light velocity $c$, respectively. Equation (29) has two real roots, $\omega_{1}^{2}$ and $\omega_{2}^{2}$, such that $\omega_{1}^{2} \omega_{2}^{2}=-k^{2} v_{t h . y}^{2}+3\left(1+k^{2}\right)$ $k^{2} v_{t h, x}^{2}$. Thus, we recover the case of the WI if one of these roots is negative, say $v_{t h, y}^{2}>3\left(1+k^{2}\right) v_{t h, x}^{2}$. We can make an estimation of the roots $\omega_{1}^{2}$ and $\omega_{2}^{2}$ by assuming $v_{t h, x / y} \ll 1$, giving $\omega_{1}^{2} \simeq 1+k^{2}$ and $\omega_{2}^{2} \simeq-k^{2}\left(v_{t h, y}^{2}-3\left(1+k^{2}\right) v_{t h, x}^{2}\right) /\left(1+k^{2}\right)$. From this last inequality, one gets the threshold condition on the temperature anisotropy necessary to initiate the instability. Furthermore, the maximum growth rate is obtained for wavevectors $k \simeq \sqrt{\varphi / \sqrt{3}-1}$, where $\varphi=v_{t h, y} / v_{t h, x}$.

\section{NUMERICAL SOLUTION OF THE MULTI-STREAM MODEL (THE R-MODEL)}

We focus here on the numerical study of the Weibel instability using the multi-stream model. Our aim is first to check the validity of the dispersion relation given by Eq. (29) in the case of an initial water-bag distribution function.

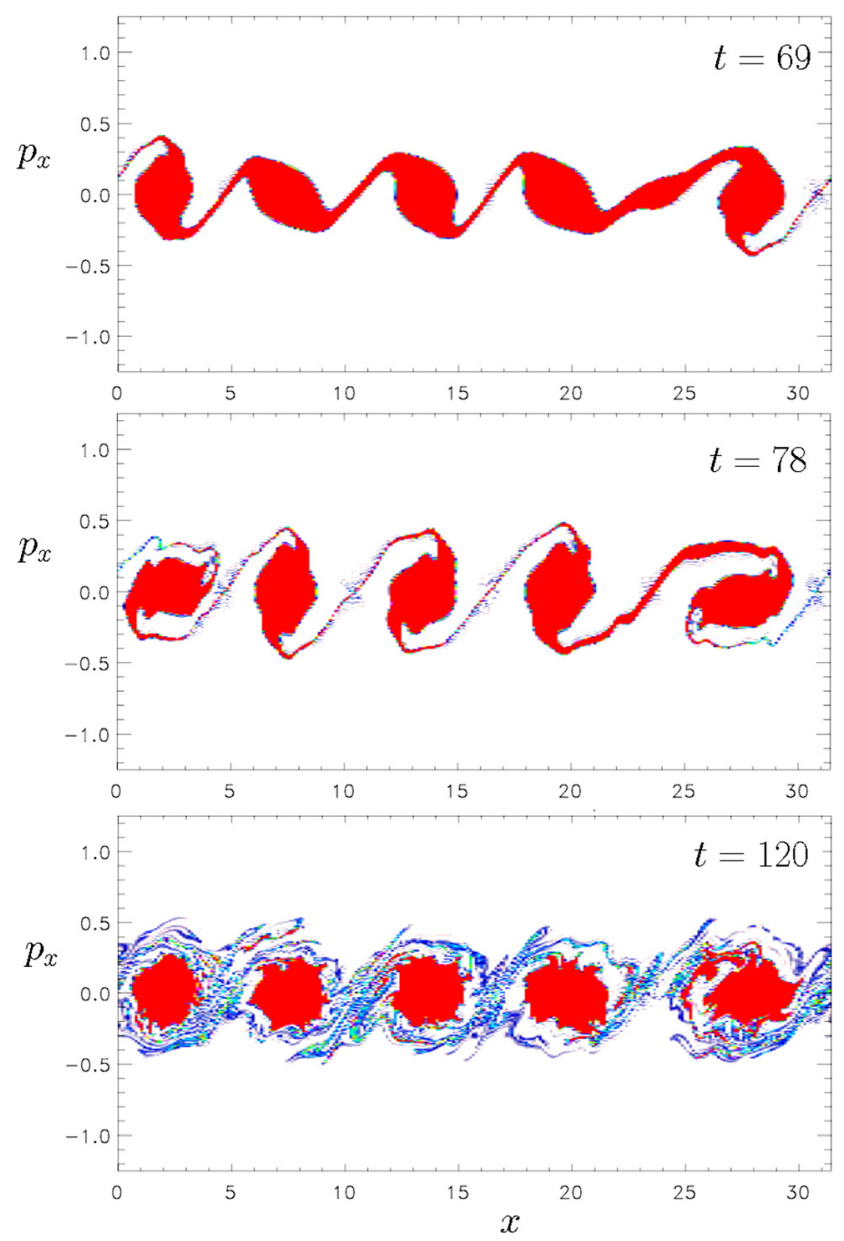

FIG. 3. The $x-p_{x}$ phase space at $t=69,78$, and 120 , same simulation as in Fig. 2. 
TABLE I. Physical parameters used in the multi-stream simulations.

\begin{tabular}{l}
\hline Physical parameters for streams \\
\hline R-code with 3 streams \\
$F_{1}=F_{-1}=\frac{1}{6}$ \\
$F_{0}=\frac{2}{3}$ \\
$C_{1}=-C_{-1}=\sqrt{3} m_{e} v_{t h, y}$ \\
$C_{0}=0$ \\
R-code with 5 streams \\
$F_{2}=F_{-2}=\frac{1}{12}$ \\
$F_{1}=F_{-1}=\frac{1}{6}$ \\
$F_{0}=\frac{1}{2}$ \\
$C_{2}=-C_{-2}=2 m_{e} v_{t h, y}$ \\
$C_{1}=-C_{-1}=m_{e} v_{t h, y}$ \\
$C_{0}=0$ \\
\hline \hline
\end{tabular}

To this goal, we integrate numerically the R-model by solving $j$-Vlasov equations given by Eq. (2) for different values of the number of streams. The numerical scheme is based on the semi-lagrangian scheme ${ }^{15}$ for the resolution of the Vlasov equation. Equation (2) must be coupled self-consistently to Eqs. (4) and (5) for the evolution of $E_{x}$ and $A_{y}$. The initial conditions for $f_{j}$ are built by determining the different values of the parameters $F_{j}$ and $C_{j}$ in Eq. (10) assuming a Maxwellian equilibrium. In other words, the equilibrium state is described by the set of parameters $\left\{\alpha_{j}, a_{j}, C_{j}\right\}$ for $-N \leq j \leq N$, corresponding to a plasma description in terms of $2 N+1$ streams, each stream having the same initial perpendicular momentum
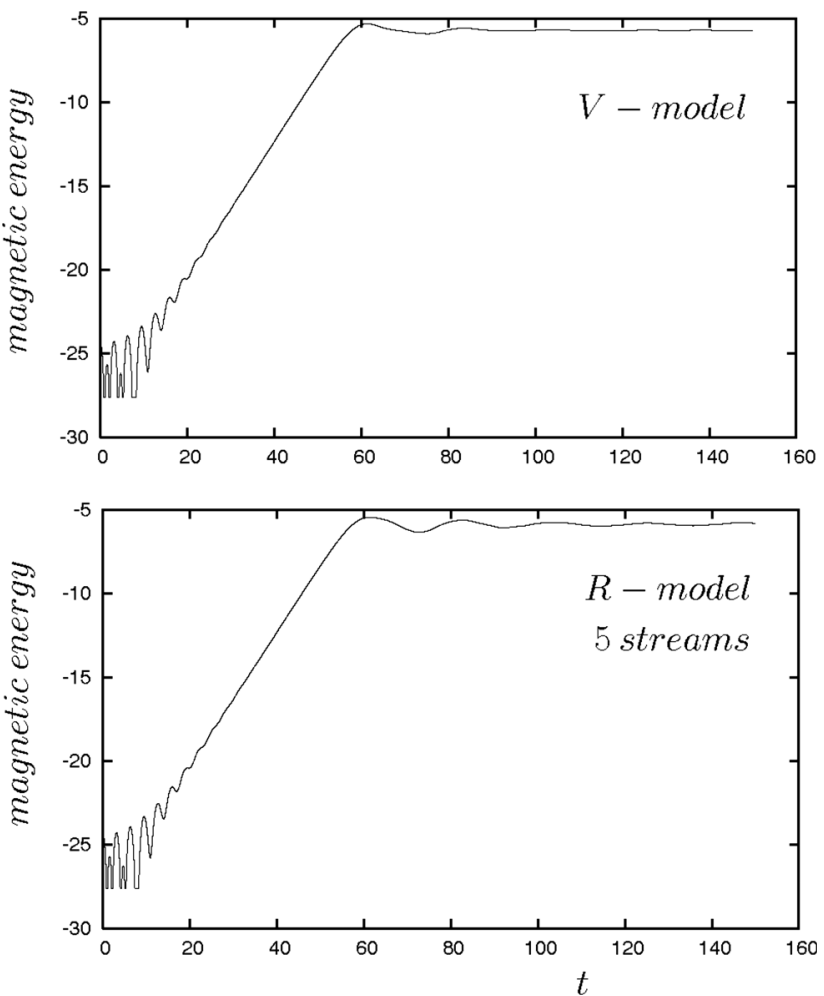

FIG. 4. Time evolution of the magnetic energy of the WI using the V-model and the R-model, top and bottom panels, respectively. The initial magnetic perturbation is monochromatic, with wave vector $k_{0}=1.75$. All physical parameters are identical for the two simulations with initial temperatures $T_{e x}=1 \mathrm{keV}$ and $T_{e y}=50 \mathrm{keV}$.
$P_{c y}=C_{j}$, constant in time. In the following, we discuss the case with $N=2$ corresponding to five streams, sufficient to describe the main kinetic effects in WI in the perpendicular momentum direction.

In summary, we use an initial water-bag shaped d.f along the longitudinal $p_{x}$ momentum direction and five streams for the description of the transverse $p_{y}$ momentum. Furthermore, the values of the set of parameters $\left\{\alpha_{j}, a_{j}, C_{j}\right\}$ are obtained through the data of $v_{t h, x}$ and $v_{t h, y}$ using Eqs. (14) and (16), and we consider the case of a symmetric distribution given by $\alpha_{-j}=\alpha_{j}, C_{-j}=-C_{j}$ and $a_{-j}=-a, a_{j}=a$ for $j>0$. By choosing $C_{0}=0$ for the central beam, $C_{1}=m_{e} v_{t h, y}$ and $C_{2}=2 m_{e} v_{t h, y}$, we get $\alpha_{0}=1 / 2, \alpha_{1}=1 / 6$, and $\alpha_{2}=1 / 12$. The Weibel instability is here driven by a temperature anisotropy of $T_{e y}=50 \mathrm{kev}$ and $T_{e x}=2.25 \mathrm{kev}$ corresponding to $a=\sqrt{3} v_{t h, x} \simeq 0.11 c$.

In the simulations, we use the electron mass and charge, the velocity of light, the plasma frequency, and the electron skin depth as characteristic quantities to normalize the equations. The electric and magnetic fields are normalized to

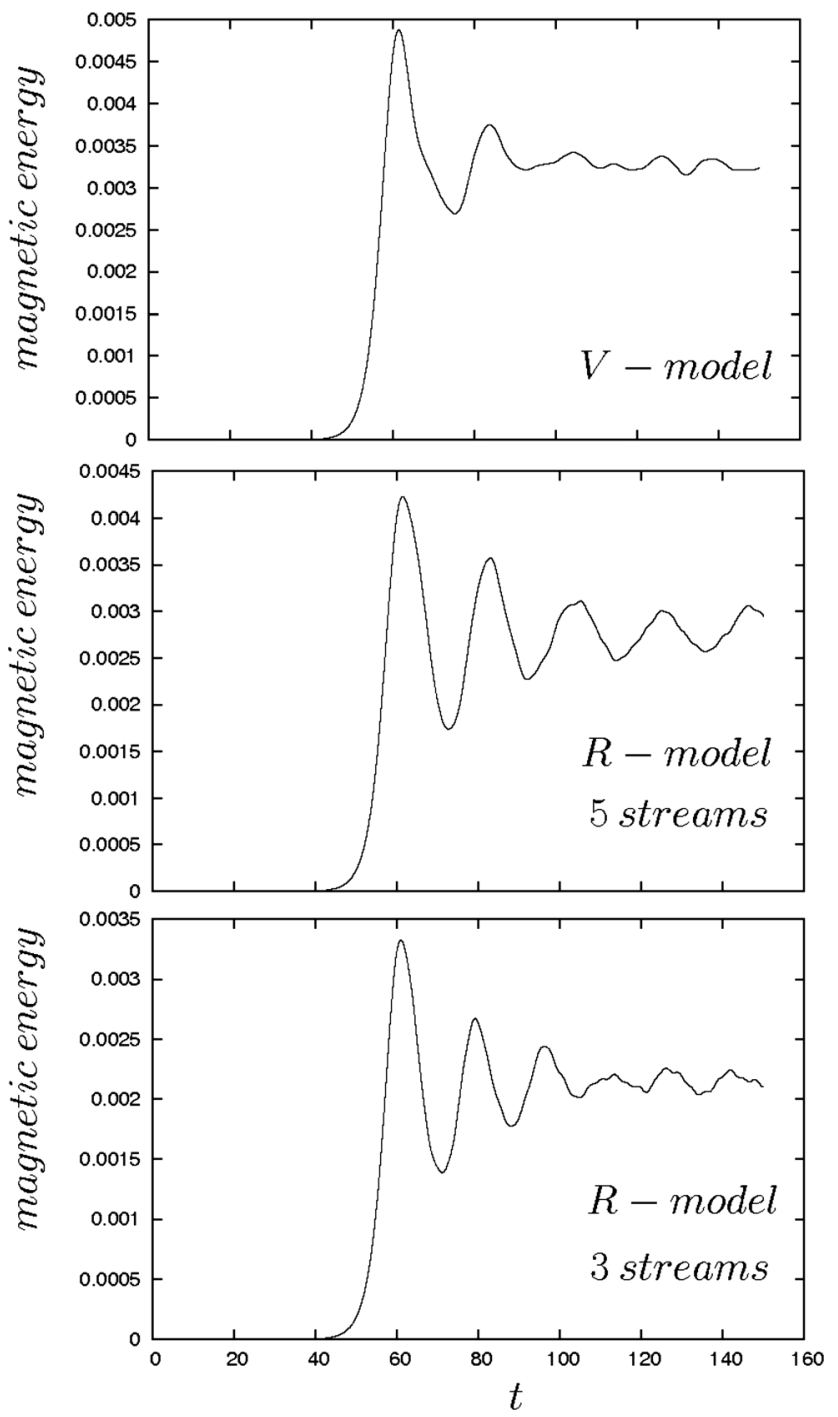

FIG. 5. Top and middle frames: Same plot as in Fig. 4 but without using a logarithmic scale. The bottom plot is obtained with the R-model, same parameters, but using only three streams. 
$\bar{E}=m_{e} c \omega_{p} / e$ and $\bar{B}=m_{e} \omega_{p} / e$. The d.f. is perturbed by magnetic fluctuations on the $B_{z}$ component of the magnetic field

$$
\delta b_{z}=\sum_{m=1}^{M} b_{0} \sin \left(2 \pi m x / L+\varphi_{m}\right),
$$

where the $\varphi_{m}$ are random phases and $b_{0}=10^{-5}$ the mode amplitude. We have taken $M=12$ modes and $L=10 \pi$. The phase space sampling used here is $N_{x} N_{p_{x}}=1025 * 513$.

In Fig. 1, we show the analytical solution of the dispersion relation, Eq. (29), by plotting the growth rate vs. the wave vector (continuous line). The crosses represent the same quantity obtained by a Fourier transform analysis of the time evolution of the different magnetic modes resulting from the numerical solution of the R-model. We see a very good agreement between the theoretical values and those obtained by the R-model.

In Fig. 2, we plot vs. time the magnetic energy $(1 / L) \int\left(B_{z}^{2} / 2 \mu_{0}\right) d x$, top panel, the inductive electric energy $(1 / L) \int\left(E_{y}^{2} / 2 \varepsilon_{0}\right) d x$, middle panel, and the electrostatic energy $(1 / L) \int\left(E_{x}^{2} / 2 \varepsilon_{0}\right) d x$, bottom panel. As expected, the magnetic energy density increases exponentially first and then saturates at $t \simeq 70$. The inductive electric field component $E_{y}$ is also amplified, but remains at much smaller amplitude. Finally, the electrostatic energy starts growing later non linearly, but remains also at very low values. During the saturation phase, $t>70$, the magnetic energy exhibits a low frequency oscillatory behavior with very a weak damping of the amplitude level. An oscillatory behavior is also visible on the electrostatic energy component, but on a much higher frequency (bottom panel), while the electromagnetic part of the electric field energy is just depleted (middle panel) without oscillating. The electrostatic activity plays a major role in the saturation phase of the Weibel instability: we observe in Fig. 2 (bottom panel) that the electrostatic energy increases strongly in the saturation regime reaching a relative maximum around $t \simeq 75$. Our multi-stream model allows a detailed examination of the particle dynamics originating the oscillatory behavior observed on both the magnetic and electrostatic energy. In Fig. 3, we show the shaded iso-contours of one of the streams (with $p_{y}=C_{1}=m_{e} v_{t h, y}$ ) in phase space at three different times. We observe the formation of five vortex-like structures, corresponding to the development of fast growing mode (see Fig. 1), $k=1$, allowed by the numerical box. At $t=69$, the last vortex, located in the region $25 \leq x \leq 30$, rotates but out of phase with respect to the other vortices. At $t=78$, this dephasing in the rotating motion is still visible. Eventually, on longer times (see, last frame at $t=120$ ), the phase space vortex structures become very similar showing, in particular, no pairing tendency at all, as one would expect instead from the corresponding phase space evolution in the electrostatic limit. During this vortex evolution with a final circle-like shaped structures,

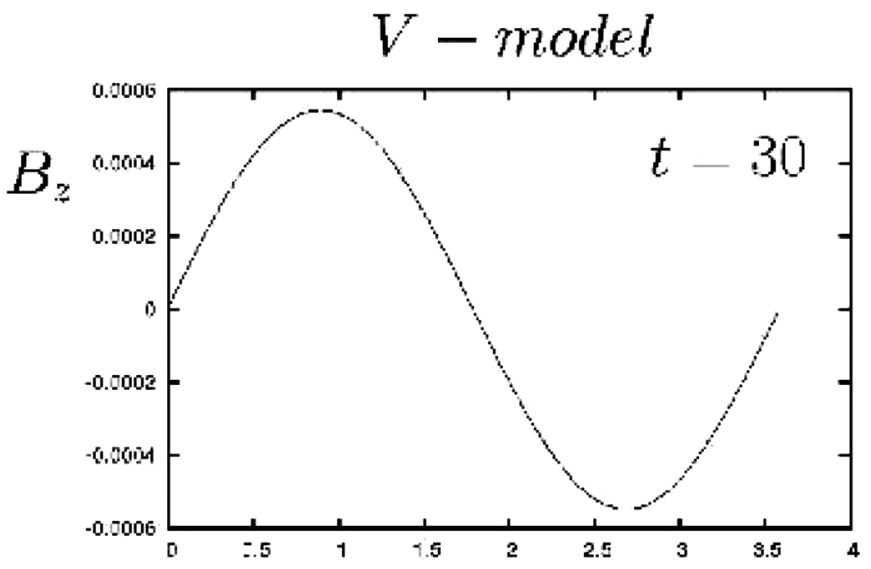

$R$-model 5 streams
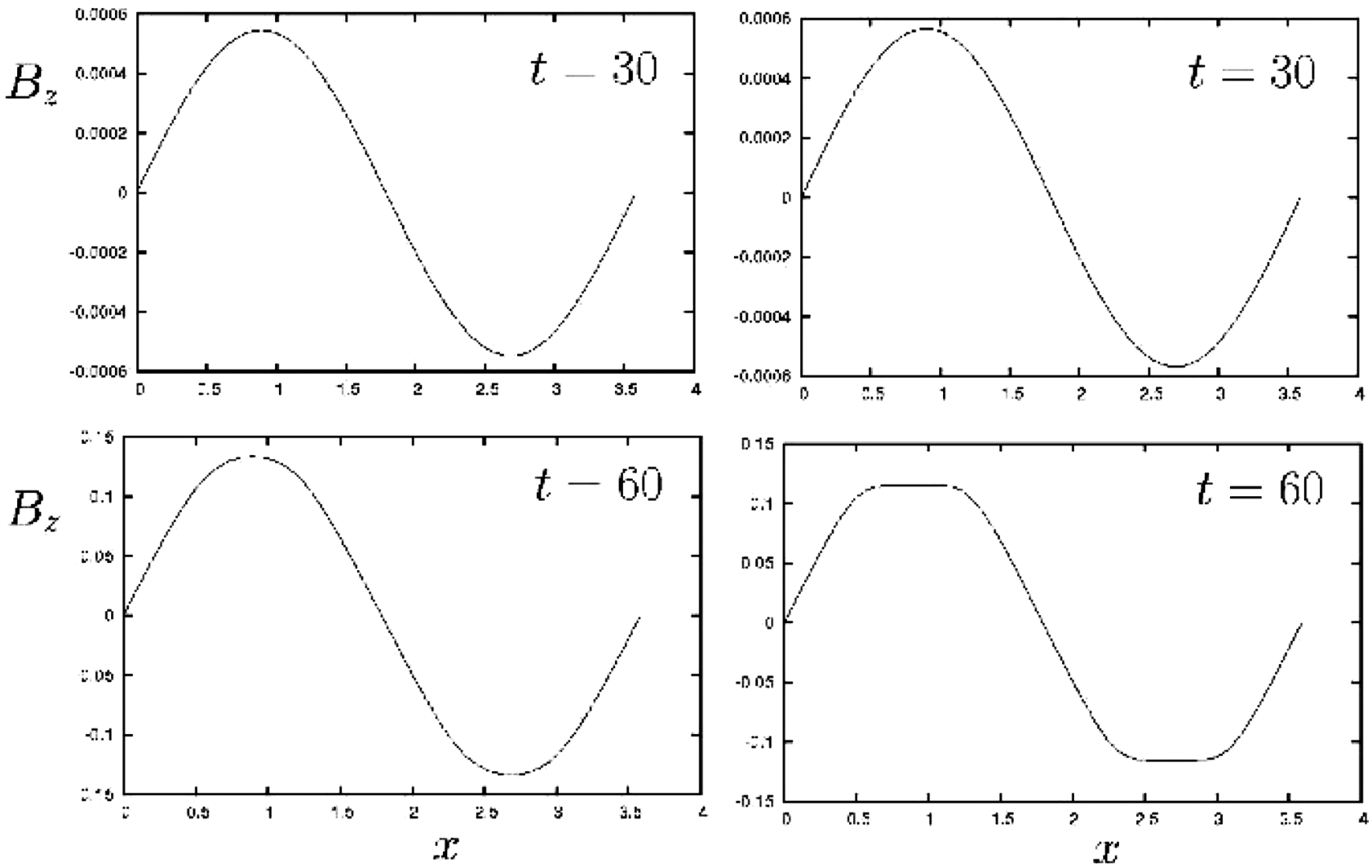

FIG. 6. The magnetic field $B_{z}$ vs. $x$ at $t=30$ and 60 using the V-model (left column) and the R-model (right column) with five streams. The bottom panels correspond to the time when the magnetic energy reaches its maximum level. The physical parameters are equal to those of Fig. 4. 
the filamentary structures is dissipated on the grid length scale corresponding to a slow decreasing of the magnetic energy, as can be seen in the top panel in Fig. 2.

\section{NUMERICAL COMPARISON BETWEEN THE FULL V-VERSION AND THE R-MODEL}

From a mathematical point of view, the multi stream model can be view as a Hamiltonian reduction technique of the phase space dimensionality, corresponding to the elimination of the perpendicular momentum $p_{y}$ that remains "hidden" in the stream $C_{j}$. Of course, an accurate description of a continuous distribution in $p_{y}$ can be obtained only using a large number of streams. However, in the case of the Weibel instability, the macroscopic description requires only the first moments of $f$, indicating that only a small number of streams is necessary for a good description of the instability even in the non linear regime (we will see in the following that five streams are required to obtain a detailed dynamics in phase space).

We now focus on the numerical study of the WI, aiming at comparing in the $1 \mathrm{D} 2 \mathrm{~V}$ limit, the multi-stream R-model (see Sec. II) and the full Vlasov-Maxwell model (hereafter
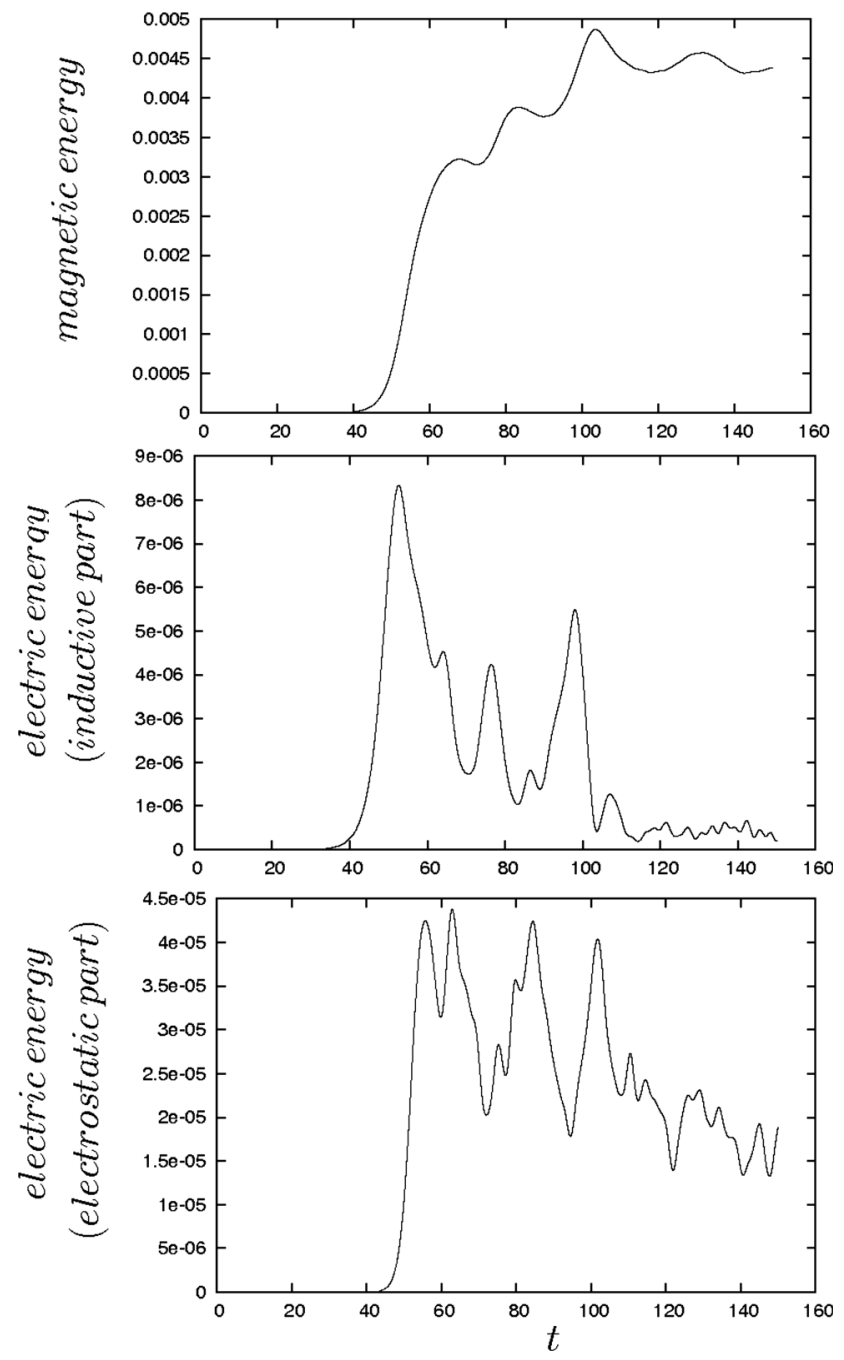

FIG. 7. Time evolution of the magnetic energy, the inductive electric energy and the electrostatic energy, and the top, middle, and bottom panels, respectively, obtained with the V-model for the WI. The initial temperatures are $T_{e x}=1 \mathrm{keV}$ and $T_{e y}=50 \mathrm{keV}$. the $\mathrm{V}$-model), this last solved by means of semi-lagrangian algorithm that integrates the Vlasov equation in the $\left(x, p_{x}, p_{y}\right)$ phase space in Ref. 15.

A set of numerical simulations of the WI has been performed by using as initial condition a temperature anisotropy distribution. In particular, for the V-model, we take a Maxwellian distribution function corresponding to $T_{e x}=1 \mathrm{keV}$ and $T_{e y}=50 \mathrm{keV}$ along the longitudinal $p_{x}$ and the perpendicular $p_{y}$ components, respectively. For the R-model, we have performed two simulations with $N=1$ and $N=2$, corresponding to three and five streams, respectively. The full set of parameters of the R-model simulations is summarized in Table I. Again, we use the electron mass $m_{e}$ and charge $e$, the velocity of light $c$, the plasma frequency $\omega_{p}$, and the electron skin depth $c / \omega_{p}$ as characteristic quantities to normalize the equations. The numerical space domain in dimensionless units is given by $L=2 \pi / k_{0}$. We choose here $k_{0}=1.75$. We perturb the system by a magnetic perturbation, $B_{z}=b_{0} \sin k_{0} x$, with $b_{0}=10^{-4}$ as initial amplitude. The phase space sampling for the V-model is $N_{x} N_{p_{x}} N_{p_{y}}=256^{3}$ while for the R-model is
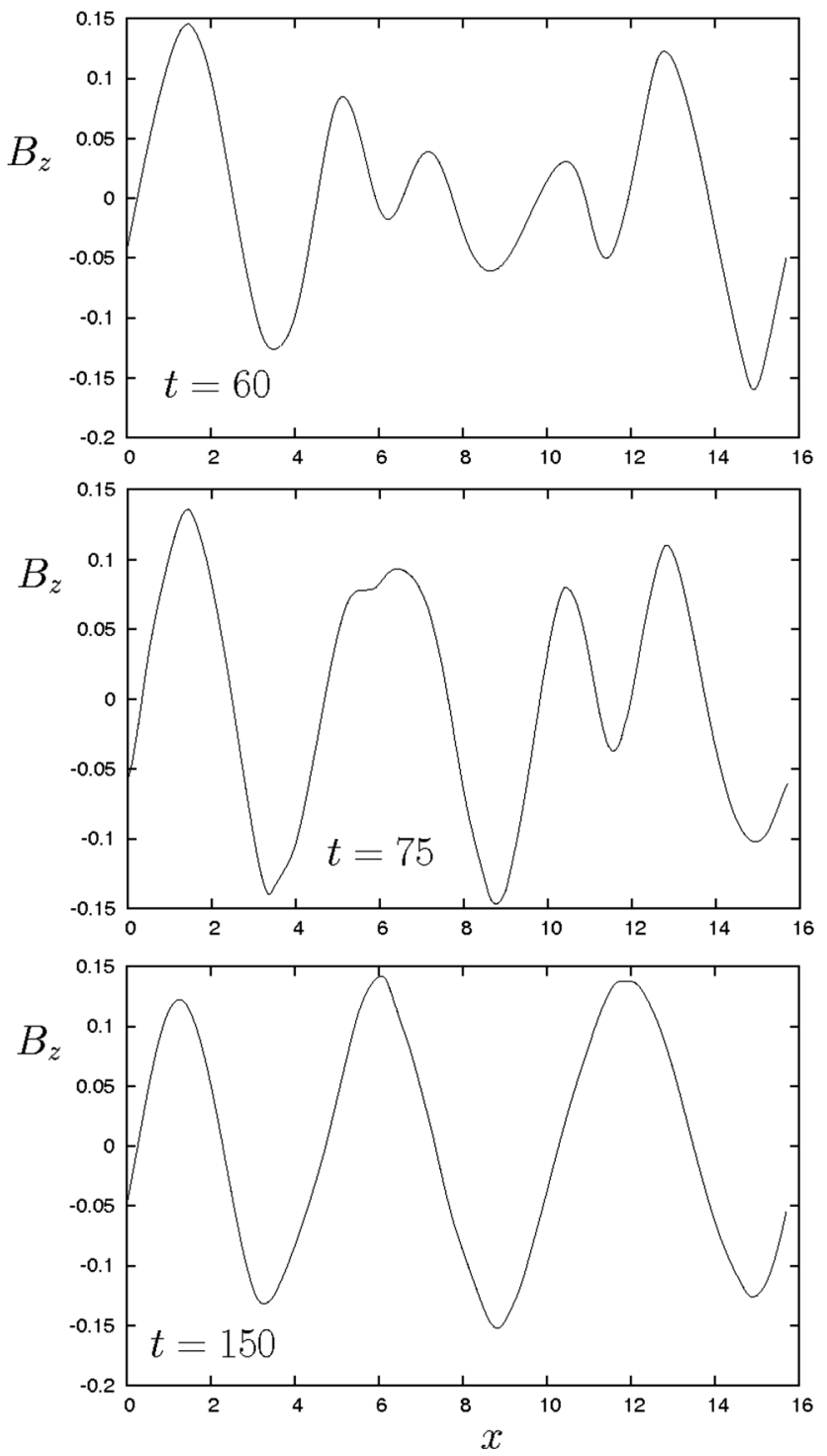

FIG. 8. The magnetic field $B_{z}$ vs $x$ at $t=60,75$, and 150 for the same simulation shown in Fig. 7. 
$N_{x} N_{p_{x}}=1024 \times 512$ grid points. The time step used in the simulations is $\delta t=0.0080$.

\section{A. Comparison in the electromagnetic regime of WI}

In Fig. 4, we show the time evolution of the total magnetic energy $\frac{1}{2 \mu_{0} L} \int_{0}^{L} B_{z}^{2} d x$ for the $\mathrm{V}$-model and the R-model (five streams), top and bottom panel, respectively. The initial perturbation is monochromatic with wave vector $k_{0}=1.75$. All the physical parameters used for the two simulations are identical. We see that the energy density of $B_{z}$ increases exponentially with the same growth rate, $\eta_{\text {num }} \simeq 0.21$, until $t \simeq 60$ and then saturates at larger times, in agreement with the results reported in Ref. 4 . The numerical growth rate is in very good agreement with the theoretical one, $\eta_{t h}=0.23$, obtained by solving the dispersion relation of the WI, Eq. (29).

On the other hand, the saturation level is a somewhat different in the two cases, as shown by Fig. 5 where we plot again the magnetic energy as in Fig. 4, but now without using a logarithmic scale, top and middle panels. In the bottom panel of Fig. 5, we plot the same quantity but obtained with the R-model using only three streams.
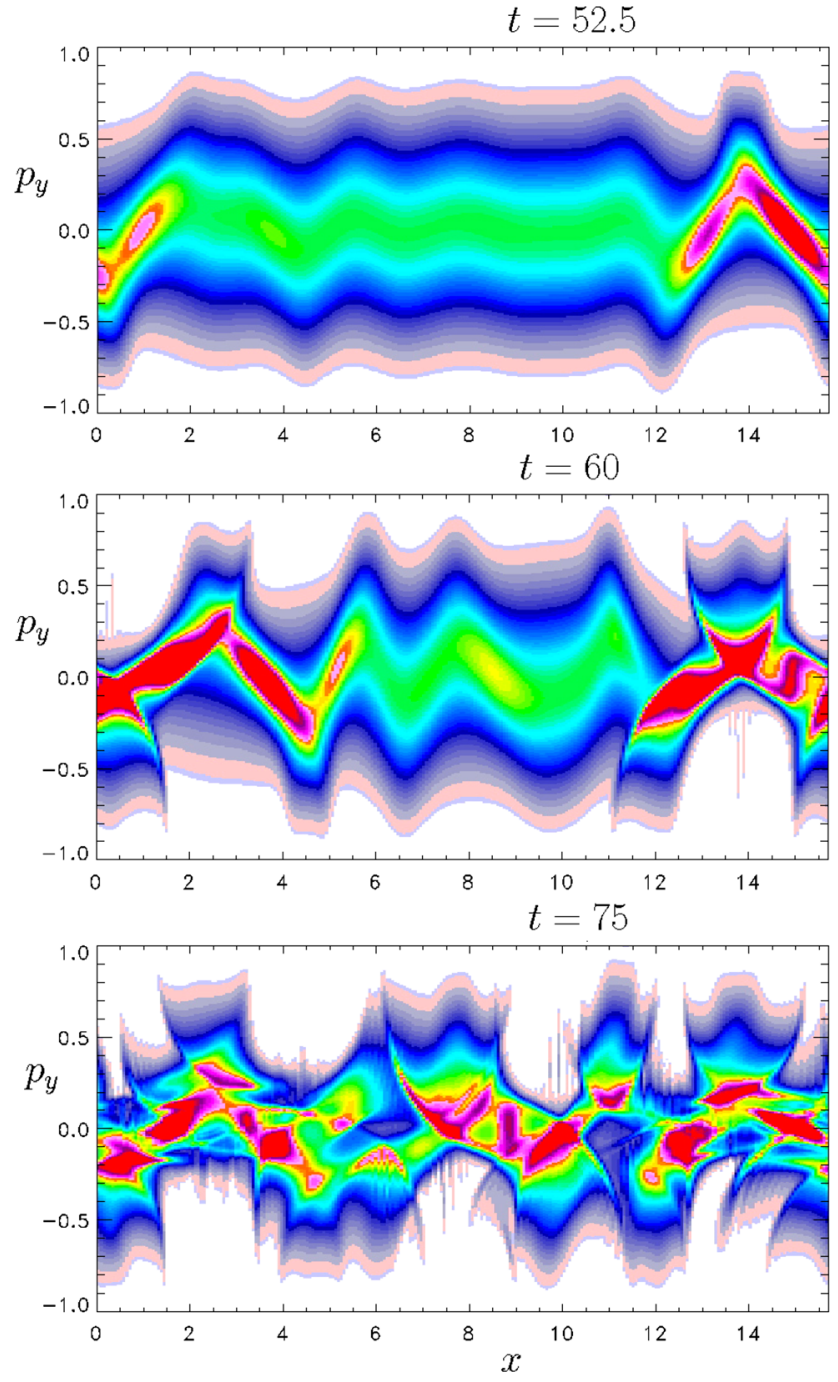

FIG. 9. The $x-p_{y}$ phase space at $t=52.5,60$, and 75 using the V-model. The physical parameters are the same as in Fig. 7.
Even if qualitatively the behavior is similar, we see that the level of saturation of the magnetic energy for the R-model with three streams, $E_{m}^{s a t}=0.0021$, is well below the value measured in the $1 \mathrm{D} 2 \mathrm{~V}$ Vlasov model, $E_{m}^{\text {sat }}=0.0032$, while an intermediate value of $E_{m}^{s a t}=0.0029$ is obtained for the R-model when using five streams. Fig. 6 displays the magnetic field component $B_{z}$ vs. $x$ at $t=30$ and $t=60$, upper and bottom panels, for the $\mathrm{V}$-model and the R-model with five streams, left and right panels, respectively. While during the linear phase the results are identically even quantitatively, we see that quantitative differences appear as soon as non linear effects come into play producing a kind of "plateau" in the regions around the field maximum and minimum, thus lowering the energy level of saturation.

\section{B. Onset of the secondary vortex-merging instability}

We now investigate the non linear coupling of the electromagnetic WI with particle dynamics along the
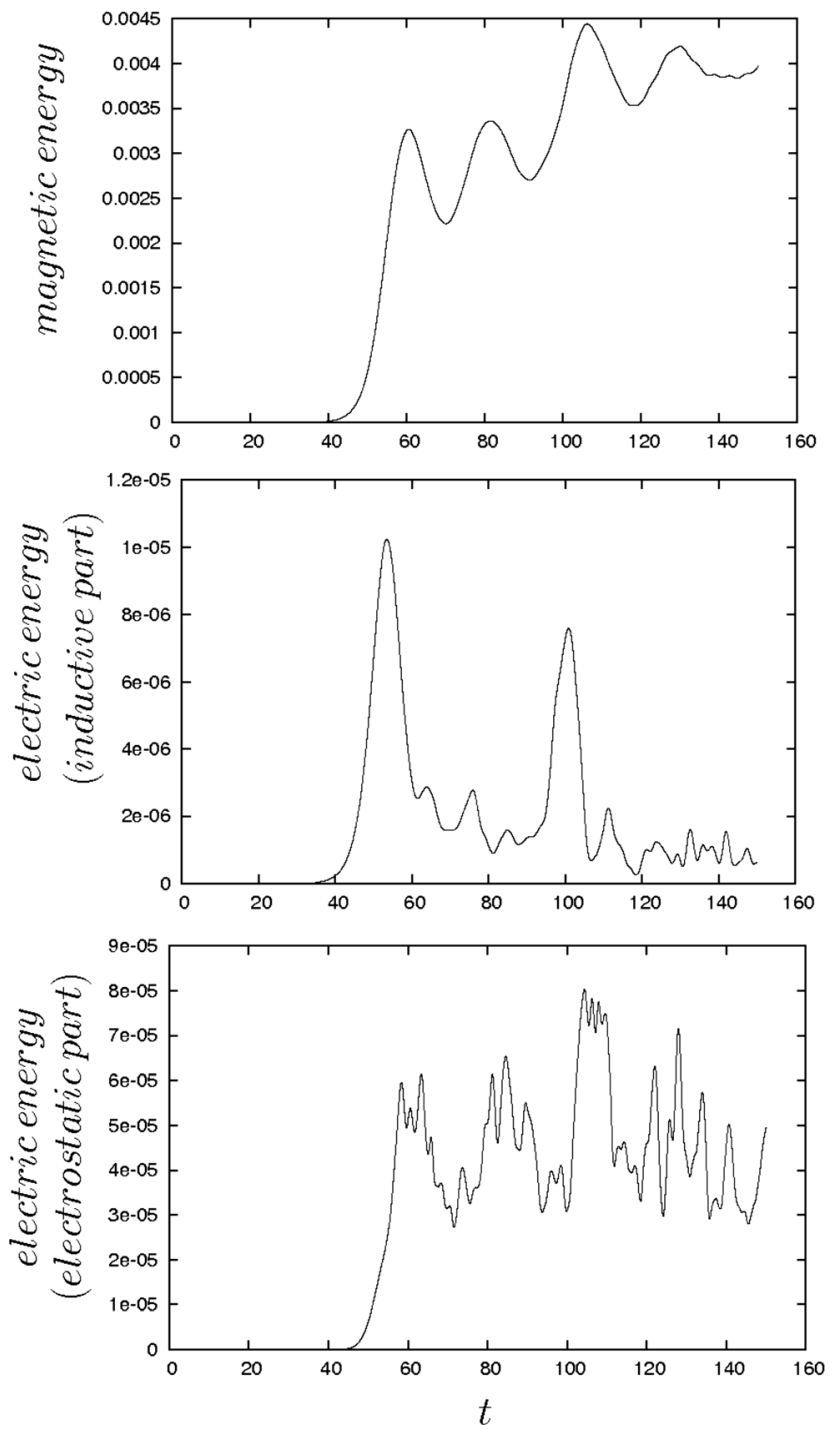

FIG. 10. Time evolution of the magnetic energy, the inductive electric energy and the electrostatic energy, and the top, middle, and bottom panels, respectively, obtained with the R-model with five streams. The physical parameters are equal to those used in the numerical simulation performed with the V-model and presented Fig. 7. 
longitudinal direction. We take the same physical parameters as in the previous cases, i.e., $T_{e x}=1 \mathrm{keV}$ and $T_{e y}$ $=50 \mathrm{keV}$ along the longitudinal and perpendicular directions, but using a larger numerical box. The spectral resolution here is $\Delta k=2 \pi / L=0.2$.

We discuss, as first, the $1 \mathrm{D} 2 \mathrm{~V}$ results obtained with the V-model using an initial perturbation on the magnetic field given by Eq. (30) still taking $M=12$ and $b_{0}=10^{-5}$. In Fig. 7, we show the growth of the magnetic, inductive electric, and electrostatic energy during the linear and the non linear phase. After the initial exponential growth, $t \leq 60$, the magnetic energy (top panel) continue to grow, but now linearly in time and with a low frequency modulation until $t \simeq 100$. A saturation phase follows for longer times. On the other hand, the energy density of the inductive electric field component, $\frac{1}{2 L} \int_{0}^{L} \varepsilon_{0} E_{y}^{2} d x$ (middle panel), rapidly decreases after the exponential growth, $t>60$, still showing a low frequency modulation characterized by local peaks. This different evolution with respect to the magnetic energy is driven by the self-reorganization of the magnetic field $B_{z}$ in terms of an inverse cascade up to the mode $m=3$. This process is outlined in Fig. 8 where we plot the spatial profile of $B_{z}$ vs. $x$ at three different times, $t=60,75$, and 150. The figure clearly shows the transition from the initial $m=5$ mode, top panel, to $m=4$, middle panel, ending to $m=3$, bottom panel. Finally, from Fig. 7, we see that the electrostatic energy, $\frac{1}{2 L} \int_{0}^{L} \varepsilon_{0} E_{x}^{2} d x$ (bottom panel) also decreases for $t>60$, but much more slowly and again showing an oscillatory low frequency behavior with strong peaks but out of phase with respect to the inductive electric energy.

In Fig. 9, snapshots of the electron distribution function in the $x-p_{y}$ phase space are shown. Once the electromagnetic fields become strong, $t \simeq 60$ (middle frame), the $E_{y}$ field component accelerates the particles along the $y$ - direction, eventually forming a layered Y-shaped structure. Although the complexity of the phase space distribution increases further with time, we see however that the modulation of $f$ is connected to the modulation of the $z$-component of the magnetic field, as shown in Fig. 8. For instance, at time $t=60$, at the maximum of the magnetic energy density, we see a modulation on the mode $m=5$. Furthermore, we observe a strong deformation of the d.f. in the region near $x \sim 2$ and $x \sim 12$, where the magnetic field reaches its maximum values. In the last frame of Fig. 9, bottom panel, we observe the d.f. after the merging of the vortices modified in order to represent now the mode $m=3$. In particular, the resulting d.f. is strongly stretched and bended, being far a Maxwellian like distribution.
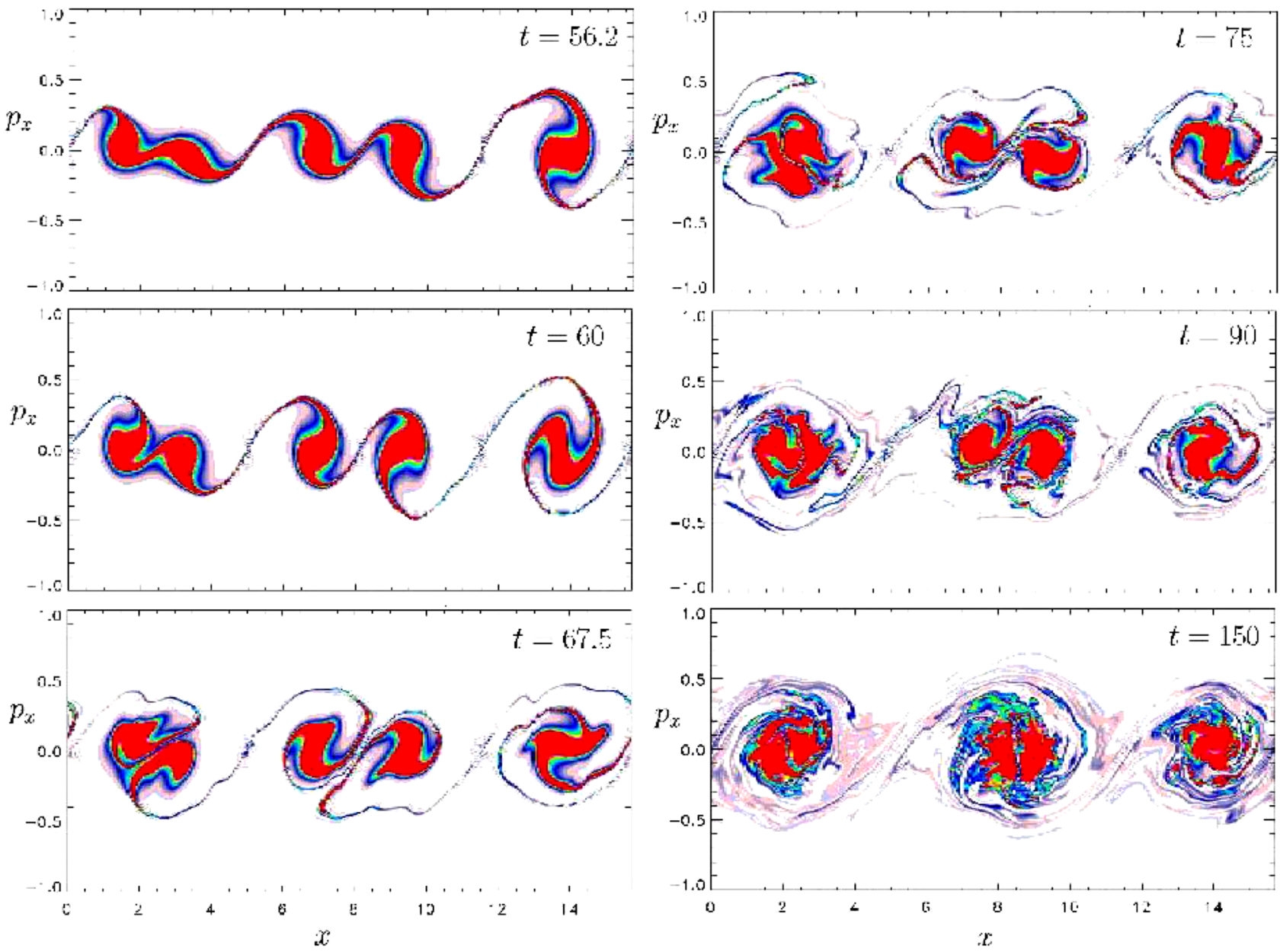

FIG. 11. The phase space $\left(x, p_{x}\right)$ for the last stream of momentum $p_{y}=2 m_{e} v_{t h, y}$ at different times using the R-model. The physical parameters are the same as those of Fig. 10 . 


\section{Detailed analysis of WI using the multi-stream model}

The analysis of the results of the WI discussed in Sec. V B was obtained by solving numerically the V-model, i.e., the full 1D2V Vlasov model. We present now the results of a numerical simulation obtained using the R-model and taking the same set of physical parameters as in the previous case. In this simulation, we have taken five streams to describe the evolution of the WI and the resulting coupling with Langmuir waves.

First of all, we see that, even with a relatively small number of streams (five in the present case), the main features of the instability are recovered. In particular, our kinetic R-model approach confirms the existence of three different phases, as shown in Fig. 10, top panel, where we plot the time evolution of the magnetic energy vs. time. In the middle and bottom panels, we plot the inductive and electrostatic contributions of the total electric energy vs. time, respectively. Generally speaking, we observe that in the non linear regime, $t>60$, the electric energy now tends to decrease much less with respect to what observed with the V-model, Fig. 7, due to less dissipative numerical effects of a reduced phase space approach. The last panel indicates a strongly oscillatory behavior of the electrostatic energy, thus showing that the electrostatic activity plays a major role during the non linear regime of the WI, in agreement with previous results (see, e.g., Refs. 4 and 7).

A very important feature of the R-model is the possibility of separating the specific contribution of each "bunch" of particles during the development of the instability. In particular, let us now focus our attention on the dynamics of the faster stream, corresponding to a value of the momentum $\left(C_{2}=2 m_{e} v_{t h, y}\right)$. In Fig. 11, we show the distribution function of the selected stream $f_{2}$ in the "reduced" phase space $\left(x, p_{x}\right)$ at six different times. As mentioned in Sec. III, the evolution of the distribution function is characterized by a differential rotation in phase space, which depends locally on the value of the magnetic field. The first panel, at the end of the linear growth of the instability, indicates that the instability has reached the saturation phase when the e.m. trapping generates five vortices emerging from the growth of the most unstable mode, $m=5$, as also observed in the magnetic field $B_{z}$. The phase space vortices evolution allows us to obtain an estimate of the rotation frequency. The unstable magnetic field perturbations grow to a sufficiently large amplitude so that the magnetic field $B_{z}$ saturates due to magnetic trapping where the frequency $\omega_{B}$ is given by, in dimensionless units, $\omega_{B}=\left[k p_{y} \omega_{c e}\right]^{1 / 2} \sim\left[k_{m=3}\left\langle p_{y}^{s a t}\right\rangle \omega_{c e}\right]^{1 / 2}$. By taking $k_{m=3}$ $=1.20,\left\langle p_{y}^{\text {sat }}\right\rangle \approx(3 / 2) v_{t h, y} \simeq 0.47$, and $\omega_{c e} \simeq 0.13$, we get
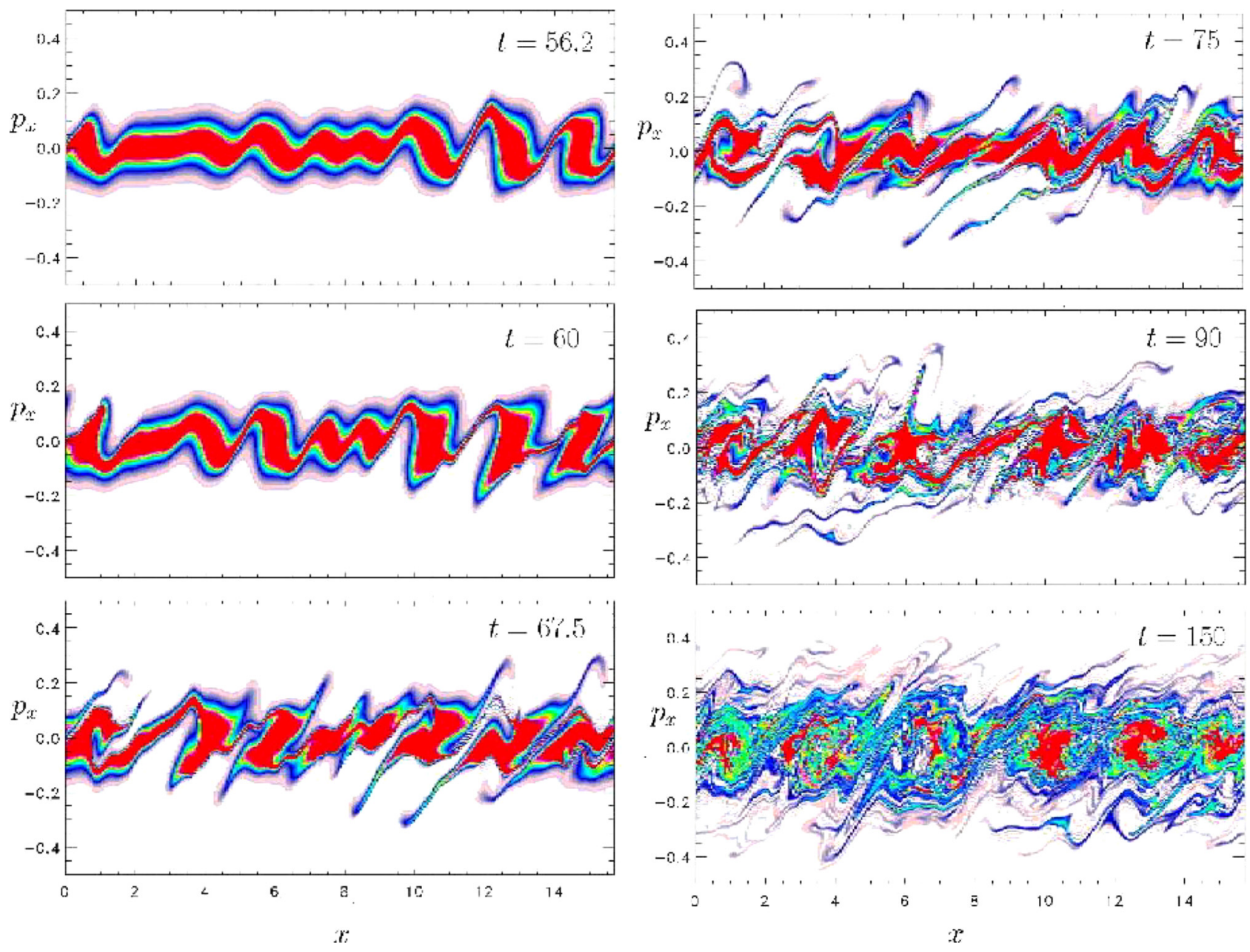

FIG. 12. The phase space $\left(x, p_{x}\right)$ as in Fig. 11 but for the central stream of momentum $p_{y}=0$. 
$\omega_{B}=0.27 \omega_{p}$. For instance, in the time interval $[56.2<t$ $<67.5]$, the last vortex structure located around $x \sim 14$, makes a half a period rotation with frequency $\omega_{r}=\pi / 11.3$ $\simeq \omega_{B} \simeq 0.278 \omega_{p}$, in good agreement with the predicted magnetic trapping frequency.

We consider now the phase space evolution of the d.f. for the fast stream, as shown in Fig. 11 by the six panels at different time instants. We observe, in agreement with the classical electrostatic picture of $1 \mathrm{D}-1 \mathrm{~V}$ phase space dynamics, an inverse cascade process of the phase space vortices occurring now in the electromagnetic regime in the framework of a 1D-2V configuration. Indeed, the vortices associated to the fast growing mode $m=5$, clearly visible at $t=56.2$ (first panel), start to pair eventually leading to four vortices, $m=4$, around $t=70$, and finally to three vortices, $m=3$, visible at $t=150$ (last time of the simulation). We note that as soon as the WI saturates, the electrostatic energy start to grow eventually leading to the coupling of the Weibel instability with Langmuir waves, in agreement with recent results obtained in Ref. 4.

We now focus on the behavior of the central beam located in the bulk of the distribution function in the momentum space since, as already discussed, the multi-stream description provides the opportunity of an accurate picture of the instability with respect to the full kinetic approach by looking at the dynamics of each single stream. In Fig. 12, we show the d.f., still in the $\left(x-p_{x}\right)$ space, at the same time instants as in Fig. 11. First of all, we note that no signature of the fast growing mode, $m=5$, is now observed since, as expected, no (magnetic) trapping occurs for the central beam. However, we can observe the second harmonic of the electromagnetic mode $m=3$ in the saturation regime of the instability. Thus, the observed modulations at $t=56.2$ and 60 result from the growth of the electrostatic $E_{x}$ field. At later times, $t=67.5$, we observe the formation in phase space of several populations of accelerated electrons that eventually take the form of jet-like structures evolving ballistically (see, the right side panels in Fig. 12). Signatures of wave-breaking process are also visible at later times. Note that such evidence of accelerated particles is much less visible on the fast-stream d.f. representation given in Fig. 11. On the other hand, it is the electrostatic field associated to the phase space structures, only visible on the fast-stream d.f., which it is responsible for the particle acceleration and wave-breaking processes observed on the central-stream d.f., Fig. 12.

\section{CONCLUSION}

The multi-stream model appears to be a valid alternative to the full Vlasov kinetic description of the Weibel instability. Interesting results have been obtained by exploiting the ability of the multi-stream model to take into account kinetic effects during the nonlinear regime.

This model, analogous to previous ones used for a driftkinetic model ${ }^{16}$ or to investigate the process of magnetic reconnection, ${ }^{17}$ is based on a reduction technique in a
Hamiltonian framework and offers thus an exact description of the plasma dynamics even with a small number of particle "bunches" (or streams) in the perpendicular direction in the momentum space. The multi-stream concept makes full use of the invariance of the canonical momentum. By approximating now the system as a finite number of "streams" or, in other words, as a summation over an ensemble of Dirac distributions, we have obtained the linear dispersion of the Weibel instability. The moment equivalence between a given continuous distribution function and a multi-stream distribution allows us to recover the notion of temperature.

From a physical point of view, many features of the Weibel instability can be recovered with only a relatively small number of streams, e.g., three or five streams, allowing a detailed kinetic analysis by studying the evolution of each stream d.f. separately. Furthermore, there is no constraint on the shape of the adopted d.f., which can be far from a Maxwellian shape. Relativistic effects, which have been neglected in the present paper, are now under consideration without any further conceptual difficulties, and will be the object of a forthcoming paper. It is important to point out that the multi-stream concept (i.e., transverse canonical momentum invariance) is not affected by adding an external longitudinal magnetic field excepted, of course, for a more complicated algebra.

\section{ACKNOWLEDGMENTS}

This work was granted access to the HPC resources of IDRIS center under the allocation 2011050050 made by GENCI (Grand Equipement National de Calcul Intensif).

${ }^{1}$ E. W. Weibel, Phys. Rev. Lett. 2, 83 (1959).

${ }^{2}$ R. L. Morse and C. W. Nielson, Phys. Fluids 14, 830 (1971).

${ }^{3}$ A. Stockem, M. E. Dieckmann, and R. Schlickeiser, Plasma Phys. Controlled Fusion 51, 075014 (2009).

${ }^{4}$ L. Palodhi F. Califano, and F. Pegoraro, Plasma Phys. Controlled Fusion 51, 125006 (2009).

${ }^{5}$ L. Palodhi F. Califano, and F. Pegoraro, Plasma Phys. Controlled Fusion 52, 095007 (2010)

${ }^{6}$ F. Califano, F. Pegoraro, S. V. Bulanov, and A. Mangeney, Phys. Rev. E 57, 7048 (1998)

${ }^{7}$ M. E. Innocenti, M. Lazar, S. Markidis, G. Lapenta, and S. Poedts, Phys. Plasmas 18, 052104 (2011).

${ }^{8}$ A. Ghizzo, B. Izrar, P. Bertrand, E. Fijalkow, M. R. Feix, and M. Shoucri, Phys. Fluids B 31, 72 (1988).

${ }^{9}$ T. W. Johnston, Y. Tyshetskiy, A. Ghizzo, and P. Bertrand, Phys. Plasmas 16, 042105 (2009).

${ }^{10}$ A. Inglebert, A. Ghizzo, T. Reveille, D. Del Sarto, P. Bertrand, and F. Califano, Euro. Phys. Lett. 95, 45002 (2011).

${ }^{11}$ A. Inglebert, A. Ghizzo, T. Reveille, D. Del Sarto, P. Bertrand, and F. Califano, Plasma Phys. Controlled Fusion 54, 085004 (2012).

${ }^{12}$ P. Morel, E. Gravier, N. Besse, R. Klein, A. Ghizzo, P. Bertrand, X. Garbet, P. Ghendrih, V. Grandgirard, and Y. Sarazin, Phys. Plasmas 14, 112109 (2007).

${ }^{13}$ D. C. DePackh, J. Electron. Control. 13, 417 (1962).

${ }^{14}$ P. Bertrand and M. R. Feix, Phys. Lett. A 28, 68 (1968).

${ }^{15}$ A. Ghizzo, F. Huot, and P. Bertrand, J. Comput. Phys. 186, 47 (2003).

${ }^{16}$ H. J. De Blank, Phys. Plasmas 8, 3927 (2001).

${ }^{17}$ T. V. Liseikina, F. Pegoraro, and E. Yu Echkina, Phys. Plasmas 11, 3535 (2004) 